\title{
Characterization of registered holographic lenses in a photopolymer compatible with the environment
}

\section{Caracterización de lentes holográficas registradas en un fotopolímero compatible con el medio ambiente}

\author{
T. Lloret ${ }^{1 *}$, , V. Navarro-Fuster ${ }^{1}$, M. G. Ramírez ${ }^{1}$, C. Neipp ${ }^{2, S}$, M. Ortuño ${ }^{2}$, \\ A. Beléndez ${ }^{2, S}$, I. Pascual ${ }^{1, S}$ \\ 1. Departamento de Óptica, Farmacología y Anatomía, Universidad de Alicante, Alicante, Spain. \\ 2. Departamento de Física, Ingeniería de Sistemas y Teoría de la Señal, Universidad de Alicante, Alicante, \\ Spain
}

(*) E-mail: tlloretlopez@gmail.com

S: SEDOPTICA member

Received: 06/08/2018 Accepted: 16/04/2019

DOI: 10.7149/OPA.52.2.51011

\begin{abstract}
:
In this work it is presented a device to obtain holographic lenses (LH) in an environmentally compatible photopolymer sensitized at the wavelength of $488 \mathrm{~nm}$. Using this device, LHs reconstructed with two lasers $(473 \mathrm{~nm}$ and $633 \mathrm{~nm}$ ), of the same focal and diameter have been obtained, with symmetrical and asymmetric experimental setups, and positive and negative focal. The image quality of these lenses is evaluated: experimentally; from the calculation of the MTF and aberrations, obtained by capturing the image of the focus (PSF) with a CCD sensor, and theoretically; by means of software. It is also proposed a method to improve the image quality of LH's that have more aberrations.
\end{abstract}

Key words: Holography, Holographic Lenses, Volume Holography, Photopolymers, Image Quality, MTF, Aberrations

\section{RESUMEN:}

En este trabajo se presenta un dispositivo para obtener lentes holográficas (LH) en un fotopolímero compatible medioambientalmente sensibilizado a la longitud de onda de $488 \mathrm{~nm}$. Utilizando este dispositivo se han obtenido LH's, reconstruidas con dos láseres $473 \mathrm{~nm}$ y $633 \mathrm{~nm}$ ), de la misma focal y diámetro, con montajes experimentales simétricos y asimétricos, y positivas y negativas. La calidad de imagen de dichas lentes se ha evaluado: experimentalmente; a partir del cálculo de la MTF y las aberraciones obtenidas capturando con un sensor CCD la imagen del foco (PSF), y teóricamente; mediante software. También se propone un método para mejorar la calidad de imagen de las LH's que tienen más aberraciones.

Palabras clave: Holografía, Lentes Holográficas, Holografía de Volumen, Fotopolímeros, Calidad de Imagen, MTF, Aberraciones.

\section{REFERENCES AND LINKS / REFERENCIAS Y ENLACES}

[1] D. Gabor, "A new microscope principle," Nature 161, 777-778 (1948).

[2] A. Beléndez, "Holografía: ciencia, arte y tecnología," Revista Brasileira de Ensino de Física 31(1), 1602 (2009).

[3] T. W. Stone, B. J. Thompson “Holographic and Diffractive Lenses and Mirrors," Spie Milestore Series 34 (1991). 
[4] V. Navarro-Fuster, M. Ortuño, S. Gallego, A. Márquez, A. Beléndez, I. Pascual, "Biophotopol's energetic sensitivity improved in $300 \mu \mathrm{m}$ layers by tuning the recording wavelength," Optical Materials 52, 111 (2016).

[5] T. Lloret, V. Navarro-Fuster, M.G. Ramírez, M. Ortuño, C. Neipp, A. Beléndez, I. Pascual, "Holographic Lenses in an Environment-Friendly Photopolymer," Polymers 10(3), 302 (2018).

[6] E. Hecht. Óptica. Addison Wesley Iberoamericana (2000).

[7] W. J. Smith, Modern Optical Engineering, McGraw-Hill (2000).

[8] A. Fernández-Oliveras, A.M. Pozo, M. Rubiño, "Comparison of spectacle-lens optical quality by modulation transfer function measurements based on random-dot patterns," Optical Engineering 49(8), 083603 (2010).

[9] A.M. Pozo, M. Rubiño, "Optical characterization of ophthalmic lenses by means of modulation transfer function determination from a laser speckle pattern," Applied Optics 44, 7744-7748 (2005).

[10] L. Carretero, A. Fimia, A. Beléndez, "Entropy-based study of imaging quality in holographic optical elements," Optics Letters 19, 1355-1357 (1994).

[11] L. Carretero, A. Fimia, A. Beléndez, "The computation and statistical analysis of aberrational diffraction patterns in holographic optical elements," Journal Optics 26, 161-174 (1995).

[12] J.N. Latta, "Computer-Based Analysis of Hologram Imagery and Aberrations. I. Hologram Types and Their Nonchromatic Aberrations," Applied Optics 10, 599-608 (1971).

\section{Introducción}

La holografía comenzó su andadura en el año 1948 en el laboratorio de una empresa de ingeniería eléctrica, en el cual Denis Gabor trabajaba en la mejora del microscopio electrónico [1]. Posteriormente el desarrollo del láser en la década de los 60, posibilitó el auge de la misma dentro del campo de la óptica. Entre sus aplicaciones cabe destacar la interferometría holográfica, la espectroscopía holográfica, la elaboración de memorias holográficas o la fabricación de elementos ópticos holográficos (EOH's) [2].

Un elemento óptico holográfico $(\mathrm{EOH})$ es aquel que se ha realizado a partir del proceso holográfico, es decir, haciendo interferir dos haces, el haz objeto (o) y el haz de referencia (r), sobre un medio de registro. Su funcionamiento está basado en la difracción de la luz por una estructura de franjas de interferencia almacenadas en el elemento. Elementos ópticos como lentes, separadores de haces, redes de difracción o filtros pueden ser generados por técnicas holográficas [3].

\section{Material de registro}

El material que se ha utilizado es un fotopolímero denominado Biophotopol [4]. Es un material cuyas mayores ventajas son la alta compatibilidad con el medio ambiente, y la escasez de toxicidad. Diferentes composiciones se han utilizado en estudios previos [5].

La solución de fotopolímero, con agua como disolvente, está formada por: acrilato sódico (AONa) como monómero polimerizable, trietanolamina (TEA) como coiniciador y plastificante, sal sódica de monofosfato 5'-riboflavina (RF) como colorante (máximo de absorción a la longitud de onda de $488 \mathrm{~nm}$ ) y polivinilalcohol (PVA) como soporte (Mw=130.000, grado de hidrólisis=87.7\%).

Inicialmente se prepara la disolución con las cantidades de cada uno de los componentes que aparece en la Tabla 1. En condiciones de laboratorio estándar $\left(\mathrm{T}=22^{\circ} \mathrm{C}\right.$ y $\left.\mathrm{H}_{\mathrm{r}}=40 \%\right)$ y con luz roja, a la cual no es sensible el material se mezclan todos los compuestos con un agitador magnético convencional durante 20 minutos. La disolución de fotopolímero es depositada por gravedad en láminas de vidrio cuadradas $\left(6.5 \times 6.5 \mathrm{~cm}^{2}\right)$. Una vez depositada la disolución, las láminas se dejan dentro de una incubadora (Climacell 111) con humedad y temperatura controladas $\left(\mathrm{H}_{\mathrm{r}}=60 \pm 5 \%\right.$ y $\left.\mathrm{T}=20 \pm 1^{\circ} \mathrm{C}\right)$. Cuando una parte del agua se ha evaporado (tiempo de secado aproximado 24 horas) el espesor "sólido" de la película es de aproximadamente $200 \mu \mathrm{m}$, y en ese momento ya se puede realizar la etapa de registro. 
ÓPTICA PURA Y APLICADA

www.sedoptica.es

TABLA 1. Cantidades de los componentes del fotopolímero.

\begin{tabular}{|c|c|c|c|}
\hline \hline PVA (\% w/v) & TEA (M) & AONa (M) & RF (M) \\
\hline \hline 15 & 0.15 & 0.34 & $1 \cdot 10^{-3}$ \\
\hline \hline
\end{tabular}

\section{Dispositivo experimental}

\section{3.a. Etapa de registro}

El esquema óptico del dispositivo experimental utilizado para la fabricación de las lentes holográficas se puede ver en la Fig. 1. Mientras que para la etapa de registro se ha utilizado un láser de Argón que emite a una longitud de onda de $488 \mathrm{~nm}$, para el control (monitorización) de la lente fabricada se ha empleado un láser de Helio-Neón sin expandir que emite a una longitud de onda de $633 \mathrm{~nm}$, a la cual no es sensible el material de registro. En la imagen se puede observar como el haz procedente del láser de Argón se divide, mediante una lámina separadora, en dos: los denominados haz objeto y haz de referencia. Tras ser filtrados espacialmente y colimados, el haz objeto pasa por una segunda lente refractiva (LR), de la que sale un haz convergente. Tanto el haz objeto como el haz de referencia interfieren en el plano en el que se coloca el fotopolímero. La focal de la LR y su distancia a la placa holográfica determinan la focal de la LH $(90 \mathrm{~mm})$ y el tamaño de la pupila de entrada $\left(\phi_{P E}\right)(12 \mathrm{~mm})$. La intensidad de registro se ha mantenido constante a 3 $\mathrm{mW} / \mathrm{cm}^{2}$ para todas las LH's.

Utilizando el montaje experimental de la Fig. 1 se han registrado LH's positivas y negativas. Se considera que una LH es negativa cuando en el proceso de registro la distancia muestra-LR es mayor que la distancia focal imagen de la LR. Por el contrario, si la distancia muestra-LR es menor que la distancia focal imagen de la LR, la LH registrada es positiva. Además, se han registrado LH's simétricas y asimétricas.

Las LH's simétricas se obtienen cuando los haces objeto y referencia poseen el mismo ángulo respecto a la normal a la superficie de la muestra. Las asimétricas se obtienen cuando los ángulos respecto a la normal a la muestra son distintos uno al otro. En total se han registrado 8 LH's como se muestra en la Tabla 2.

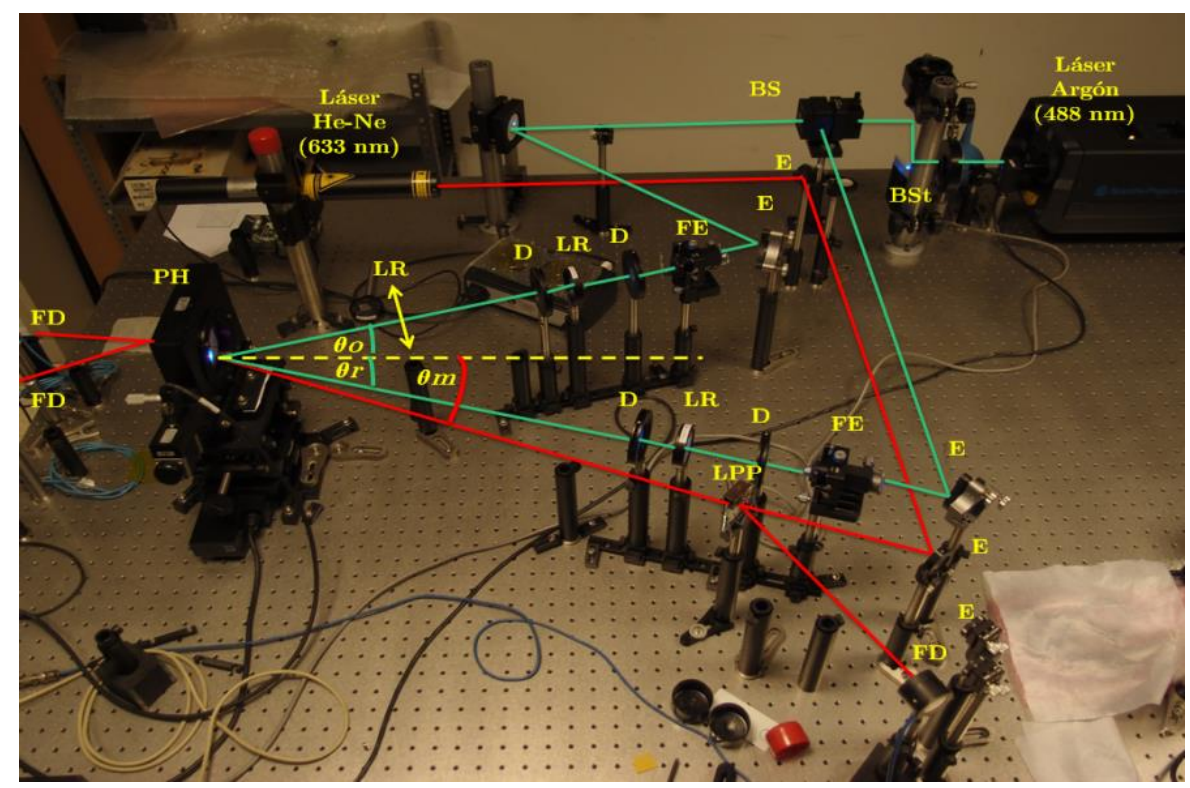

Fig. 1. Dispositivo experimental utilizado en la etapa de registro (registro simétrico). El registro se ha realizado mediante un láser Argón (488 nm) y la monitorización, mediante un láser Helio-Neón (633 nm), donde BS (divisor de haz), BSt (set de dos espejos), E (espejo), FE (filtro espacial), D (diafragma), LR (lente refractiva), FD (fotodetector), LPP (lámina plano-paralela) y PH (placa holográfica). Fuente: Laboratorio Holografía, Universidad de Alicante.

En el registro de las LH's simétricas los ángulos de referencia $\left(\theta_{\mathrm{r}}\right)$, objeto $\left(\theta_{\mathrm{o}}\right)$ y de monitorización $\left(\theta_{\mathrm{m}}\right)$ empleados son $17.1^{\circ},-17,1^{\circ}$ y $22.4^{\circ}$, respectivamente. Para las lentes asimétricas $\theta_{\mathrm{r}}, \theta_{\mathrm{o}}$ y $\theta_{\mathrm{m}}$ son $0^{\circ}, 34.2^{\circ} \mathrm{y}$ $46.8^{\circ}$, respectivamente. 


\section{3.b. Etapa de reconstrucción}

La reconstrucción de las LH's y la obtención de la PSF se realiza con un dispositivo experimental que puede verse en la Fig. 2. El haz láser empleado se filtra espacialmente y se coloca incidiendo sobre la LH con un ángulo de reconstrucción $\theta_{C}$ de 16.5 y $22.4^{\circ}$ para el caso simétrico a 473 y $633 \mathrm{~nm}$, respectivamente; y de 33.0 y $46.8^{\circ}$ para el caso asimétrico a 473 y $633 \mathrm{~nm}$, respectivamente.

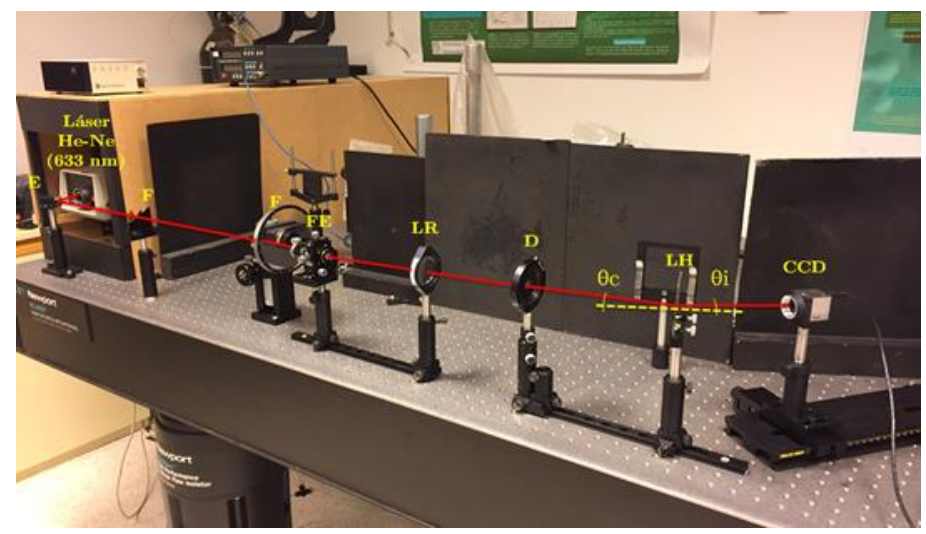

Fig. 2. Dispositivo experimental utilizado en la etapa de reconstrucción (en este caso, láser $633 \mathrm{~nm}$ ) y obtención de la PSF con la cámara CCD, donde E (espejo), FE (filtro espacial), F (filtro), D (diafragma), LR (lente refractiva), LH (lente holográfica), CCD (s ensor) y $\theta c$ y $\theta \mathrm{i}$ son los ángulos de reconstrucción e imagen, respectivamente.

\section{Evaluación de la calidad de imagen}

\section{4.a. Evaluación de la MTF}

Una de las principales herramientas para la evaluación de la calidad de imagen de una lente es el cálculo de la MTF. La MTF es una medida de la relación del contraste de la imagen respecto al contraste del objeto [6].

$$
M T F=\frac{M_{i}}{M_{o}}
$$

donde $M_{i}$ y $M_{o}$ representan los contrastes imagen y objeto, respectivamente. A su vez, el contraste, también llamado profundidad de modulación, se define como:

$$
M=\frac{I_{\text {máx }}-I_{\text {mín }}}{I_{\text {máx }}+I_{\text {min }}}
$$

Para un sistema óptico perfecto, es decir libre de aberraciones, en el que únicamente se ha tenido en cuenta el límite impuesto por la difracción según el criterio de resolución de Sparrow y en el que se considera abertura circular, se puede definir la MTF cómo [7]:

$$
M T F=\frac{2}{\pi}(\phi-\cos \phi \cdot \sin \phi)
$$

donde

$$
\phi=\cos ^{-1}\left(\frac{\lambda \cdot v}{2 \cdot A N^{\prime}}\right)
$$

$v$ es la frecuencia en ciclos por milímetros, $\lambda$ es la longitud de onda en milímetros y $A N^{\prime}$ es la apertura numérica. La frecuencia de corte límite $\left(v_{0}\right)$, es decir, la v para la cual la MTF es igual a cero, viene dada por:

$$
v_{0}=\frac{2 \cdot A N^{\prime}}{\lambda}=\frac{1}{\lambda \cdot(f / \#)}
$$

donde $f / \#$ es el número de diafragma que viene definido por:

$$
f_{/ \#}=\frac{f^{\prime}}{\phi_{P E}}
$$

siendo $f$ la focal de la lente y $\phi_{P E}$ el diámetro de la pupila de entrada. 
Es interesante notar que la Ec. 5 expresa (en términos de frecuencia espacial) el hecho de que la resolución aumenta al aumentar la apertura numérica o al disminuir la longitud de onda.

Por otra parte, el cálculo de la MTF experimental, se ha realizado a través de la PSF obtenida en la etapa de reconstrucción. La PSF (Point Spread Function) es la función de distribución de intensidad en el plano imagen cuando el objeto es puntual. En los casos en los que el sistema óptico sea perfecto y la pupila sea circular, la imagen vendrá dada por el disco de Airy. Sin embargo, en los casos en los que el sistema presente aberraciones, la PSF dependerá de dichas aberraciones.

Suponiendo que la distribución de amplitud en la imagen se puede aproximar a partir de la distribución de intensidades de la imagen $I\left(x^{\prime}, y^{\prime}\right)$ capturada con la CCD, se define la función de transferencia coherente (CTF) como la transformada de Fourier bidimensional normalizada a la distribución de intensidades según la siguiente ecuación:

$$
H\left(f_{x}, f_{y}\right)=\frac{\iint_{-\infty}^{+\infty} I\left(x^{\prime}, y^{\prime}\right) e^{-j 2 \pi\left(f_{x} x^{\prime}+f_{y} y^{\prime}\right)} d x^{\prime} d y^{\prime}}{\iint_{-\infty}^{+\infty} I\left(x^{\prime}, y^{\prime}\right) d x^{\prime} d y^{\prime}}
$$

Si consideramos una imagen de $N$ x $M$ píxeles y dimensiones $a$ x $b$ podemos considerar nuestra posición $(x$, $y^{\prime}$ ) en función de la dimensión física de la imagen. Teniendo en cuenta esto y sustituyendo las integrales de (7) por sumatorios nos quedaría:

$$
H\left(f_{x}, f_{y}\right)=\frac{\sum_{p=0}^{N} \sum_{q=0}^{M} I(p, q) \cdot e^{-j 2 \pi f_{x} x_{p}} \cdot e^{-j 2 \pi f_{y} y_{p}}}{\sum_{p=0}^{N} \sum_{q=0}^{M} I(p, q)}
$$

Según Rubiño et. al [8,9] la MTF total viene dada en función de la lente y la cámara CCD a través de la siguiente relación:

$$
M T F_{\text {total }}=M T F_{C C D} \cdot M T F_{L H}
$$

El detector CCD empleado para la captura de las PSF's está compuesto por 1360 x 1420 píxeles (horizontal $\mathrm{x}$ vertical) con una distancia entre centros de $4.65 \mu \mathrm{m}$ en ambas direcciones. Así pues, entre los fotoelementos de una determinada dirección $\Delta x$, la frecuencia de Nyquist viene definida como:

$$
\xi_{N y}=\frac{1}{2 \Delta x}
$$

cuyo valor en nuestro caso es de 107.53 líneas/mm. Esto indica que la cámara CCD Thorlabs DCU224C no es capaz de detectar frecuencias espaciales mayores de 107.53 líneas $/ \mathrm{mm}$, por lo que las MTF's de las LH's fabricadas sólo tienen validez para frecuencias de corte menores a la de Nyquist. Por otro lado, hay que destacar que en realidad las MTF's de las LH's representadas tienen implícitas la MTF de la CCD, es decir, lo que en realidad se ha representado han sido las MTF's totales.

\section{4.b. Evaluación de las aberraciones}

La evaluación de las aberraciones se puede realizar tanto en el plano de la pupila de salida, como en el plano focal imagen.

Para calcular las aberraciones en el plano focal imagen, asumimos que el fotopolímero está situado en el plano XY y que el punto fuente $Q\left(x_{q}, y_{q}, z_{q}\right)$ de una onda. Con $q=r, o, c$ y $i$ que denotan los puntos de referencia, objeto, reconstrucción e imagen, respectivamente [10,11].

El primer paso ha consistido en calcular la distancia del holograma a la imagen gaussiana $\left(R_{i}\right)$ y el ángulo imagen $\left(\alpha_{i}\right)$, es decir, el ángulo que forma el punto imagen gaussiano respecto a la normal a un plano $\mathrm{z}=0$ (plano en el que está situado el fotopolímero, ver Fig. 3).

La distancia a la imagen gaussiana viene dada por la siguiente expresión:

$$
\frac{1}{R_{i}}=\frac{1}{R_{c}}+\mu\left(\frac{1}{R_{o}}-\frac{1}{R_{r}}\right)
$$

donde $R_{r}$ y $R_{c}$ son las distancias de los haces de referencia y de reconstrucción, respectivamente, y en este caso ambas son infinito. Por otro lado, $R_{o}$ es la distancia objeto, e indica a qué distancia se encuentra el punto objeto del holograma. En el registro de LH's positivas $R_{0}$ es positivo, y en lentes negativas $R_{0}$ es negativo. 
El factor $\mu$ indica la relación entre la longitud de onda de reconstrucción y la objeto:

$$
\mu=\frac{\lambda_{c}}{\lambda_{o}}
$$

El cálculo del ángulo imagen se hace a través de la siguiente relación:

$$
\operatorname{sen} \alpha_{i}=\operatorname{sen} \alpha_{c}+\mu\left(\operatorname{sen} \alpha_{o}-\operatorname{sen} \alpha_{r}\right)
$$

De acuerdo con [8] la fase de la aberración en el punto $(x, y)$ viene dada por:

$$
\Delta(x, y)=\phi_{c}(x, y)-c(x, y) \pm\left[\phi_{o}(x, y)-\phi_{r}(x, y)\right]
$$

donde el \pm indica los primeros ordenes positivos y negativos de difracción y $\phi_{q}$ es la fase de la onda esférica. Este valor en el plano del holograma viene dado por $[10,12]$ :

$$
\phi_{q}(x, y)=\frac{2 \pi}{\lambda_{q}}\left[r_{q}(x, y)-R_{q}\right]
$$

donde $r_{q}$ es la distancia de un punto $q$ situado enfrente del holograma a un punto arbitrario de la superficie del holograma y $R_{q}$ la distancia del punto $q$ al centro del holograma.

La aberración de fase $\Delta$ se relaciona con la aberración de onda $W$ mediante la siguiente expresión:

$$
\Delta=\frac{2 \pi}{\lambda_{c}} W
$$

Usando las ecuaciones (14) y (15) y con (11), (12) y (13) se puede escribir la aberración de onda como:

$$
W=r_{c}-r_{i} \pm \mu\left(r_{o}-r_{r}\right)-\left[R_{c}-R_{i} \pm \mu\left(R_{o}-R_{r}\right)\right]
$$

La intensidad en el plano imagen en un plano normal al rayo principal (eje Z') a la distancia $z^{\prime}$ del centro de la LH (ver Fig. 3) puede escribirse como:

$$
I\left(x^{\prime}, y^{\prime} ; z^{\prime}\right)=\frac{1}{B^{2}}\left|\iint_{S} A(x, y) \exp \left[i \Delta\left(x, y ; x^{\prime}, y^{\prime} ; z^{\prime}\right)\right] d x d y\right|^{2}
$$

donde $A(x, y)=1$ (amplitud uniforme), $S$ representa el área de la pupila de salida donde se realiza la integración y $B$ es la amplitud del punto imagen gaussiano $\left(x^{\prime}=y^{\prime}=0\right)$ en ausencia de aberraciones.

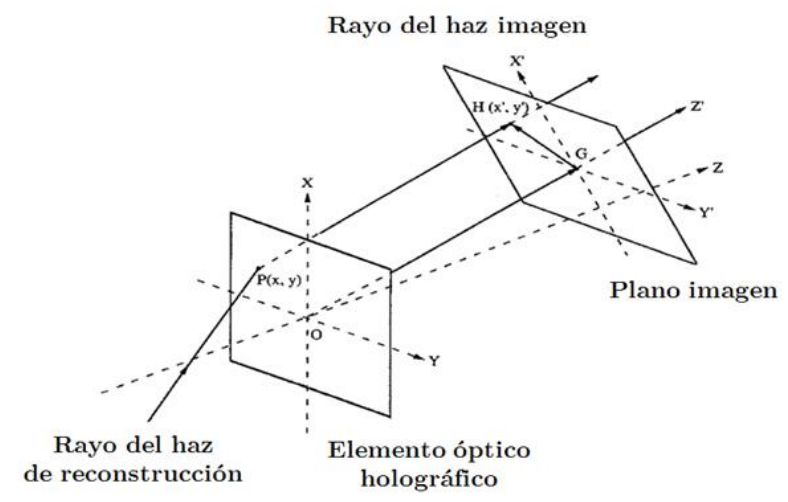

Fig. 3. Geometría de cálculo entre plano del EOH y plano imagen [12].

Por otro lado, para evaluar las aberraciones en el plano de la pupila de salida se han empleado las ecuaciones (11) a (13) descritas por Latta [12].

\section{Resultados}

\section{5.a. MTF}

En la Fig. 4 se han representado las MTF's teóricas y experimentales de las distintas LH's fabricadas en función de $v$ para distintas $\lambda_{c}$. En ausencia de aberraciones, el valor máximo de la MTF (3) viene 
determinado por la difracción. La existencia de aberraciones produce un descenso de la curva MTF frente a la v. Por consiguiente, el rendimiento de un sistema se puede obtener comparando la MTF experimental respecto a la MTF limitada por difracción cuando se tienen la $\lambda$ y $f / \#$ (5).

Según la bibliografía [3], el criterio de resolución de Lord Raigleigh predice que a una $\lambda$ menor se obtiene mayor resolución, por tanto, una mayor MTF. Para un valor de MTF de 0.1 se puede observar que las frecuencias son siempre mayores en el caso de las lentes reconstruidas con el $473 \mathrm{~nm}$. Sin embargo, para la LH asimétrica negativa se alcanza una frecuencia mayor para el $633 \mathrm{~nm}$, contrario a lo esperado. Por otro lado, también se puede observar que, el hecho de que el objeto este situado en eje (caso asimétrico) o fuera de eje (caso simétrico) también afecta a la $v$ de dichas LH's, siendo esta mayor en el caso asimétrico.

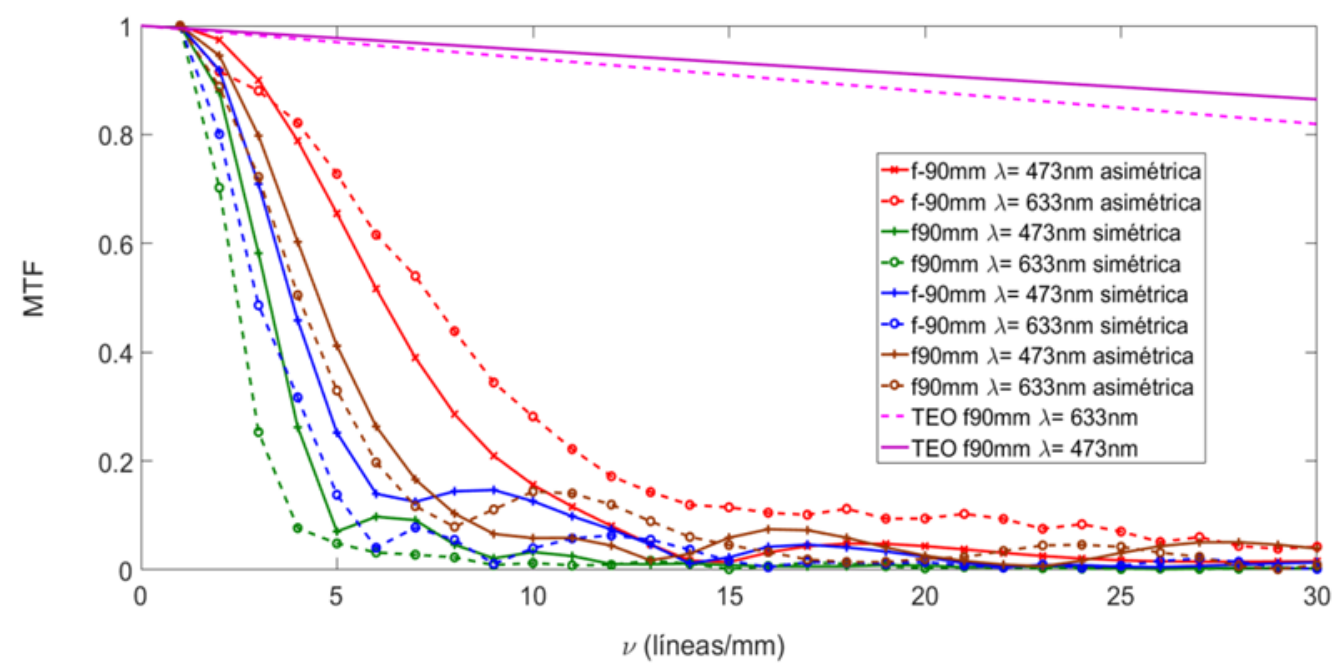

Fig. 4. Representación de las MTF's de las LH's positivas y negativas, registradas simétrica y asimétricamente. La frecuencia espa cial (u) está acotada superiormente por la frecuencia de Nyquist. Para la MTF se considerará una cota inferior de 0.1 para poder realizar una correcta estimación de los resultados obtenidos.

Los valores máximos alcanzados han sido de 17 líneas/mm en el caso de la LH asimétrica negativa reconstruida con $633 \mathrm{~nm}$ y de 12 líneas/mm si reconstruimos con $473 \mathrm{~nm}$.

\section{5.b. Aberraciones}

En el plano de la pupila de salida se han estudiado las aberraciones teóricas utilizando un script programado en MATLAB con las ecuaciones de las desviaciones del frente de onda respecto a la esfera gaussiana descritas por Latta [12].

En el plano focal imagen se han estudiado las aberraciones teóricas calculando el perfil de intensidad teórico normalizado al plano imagen gaussiano, Ec. 18. Este perfil de intensidad equivale a la métrica Razón de Strehl e indica de manera cualitativa la calidad de imagen de dichas LH's. Ya que la integral sólo se puede abordar mediante cálculo numérico se ha empleado un script programado en MATLAB correspondiente al método empleado por L. Carretero et al. [11]. Para ello, se ha supuesto que la pupila de la lente es circular, de superficie $S$ y se ha dividido en subdominios de integración.

En el estudio experimental de las aberraciones en el plano focal imagen se han utilizado las PSF's capturadas con la CCD (empleadas también para el cálculo de la MTF de las LH's) representando dicho perfil de intensidades de forma tridimensional.

En este trabajo sólo se han representado las LH's con peor calidad de imagen (LH's simétricas positivas) y las LH's con la mejor calidad de imagen (LH's asimétricas negativas).

En la Fig. 5 se han representado las LH's simétricas positivas reconstruidas con $633 \mathrm{~nm}$ (Fig. 5a, 5b y 5c) y reconstruidas con $473 \mathrm{~nm}$ (Fig. 5d, 5e y 5f) observándose menor aberración para la longitud de onda de $473 \mathrm{~nm}$. Mientras que las LH's asimétricas negativas se han representado en la Fig. 6, reconstruidas con 633 nm (Fig. 6a, 6b y 6c) y reconstruidas con $473 \mathrm{~nm}$ (Fig. 6d, 6e, 6f). Aquí se observa una mayor intensidad normalizada al plano imagen gaussiano (Fig. 6d) para la longitud de onda de $473 \mathrm{~nm}$, siendo las aberraciones en el plano de la pupila de salida y la PSF tridimensional similares. Las figuras a y d de Fig. 5 y Fig.6 
representan la aberración total ( $\left.\Delta_{\text {Total }}\right)$ en el plano de la pupila de salida. Las Fig. 6b y 6e representan los perfiles de intensidad experimentales (en u.a.). Las Fig. 6c y $6 \mathrm{f}$ representan los perfiles de intensidad teóricos normalizados al plano imagen gaussiano.

a)

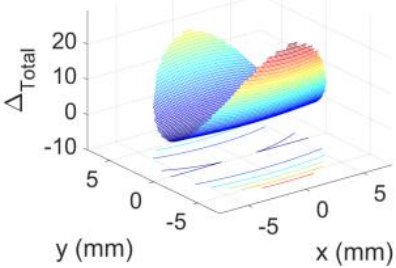

d)

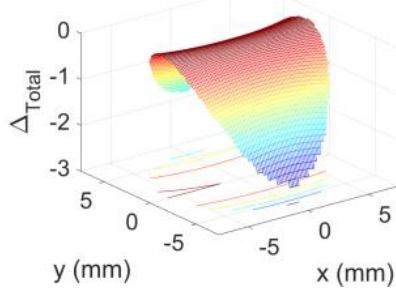

b) $y(m m)$

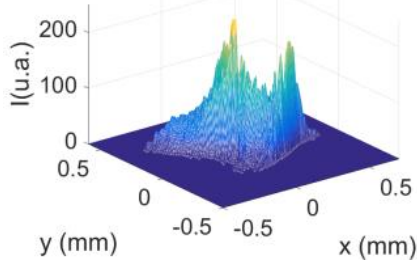

c)

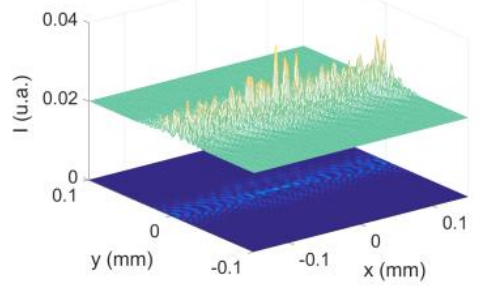

e)
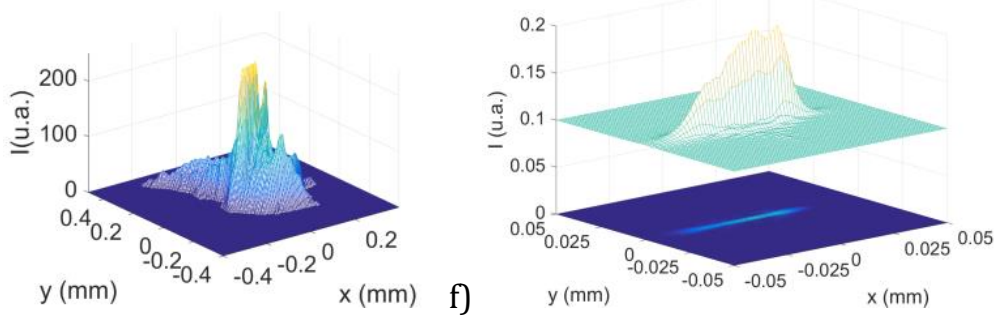

Fig 5. LH's simétricas positivas reconstruidas con $633 \mathrm{~nm}$ (Fig. 5a, 5b y 5c) y $473 \mathrm{~nm}$ (Fig. 5d, 5e y 5f). Las Fig. 5a y $5 \mathrm{~d}$ representan la aberración total en el plano de la pupila de salida (en unidades arbitrarias), las Fig. 5b y 5e representan los perfiles de intensidad experimentales (en u.a.) y las Fig. 5 c y 5 f representan los perfiles de intensidad teóricos normalizados al plano imagen gaussiano.

a)

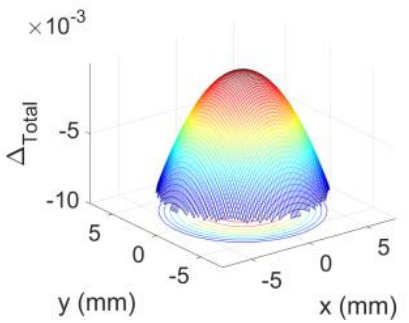

d)

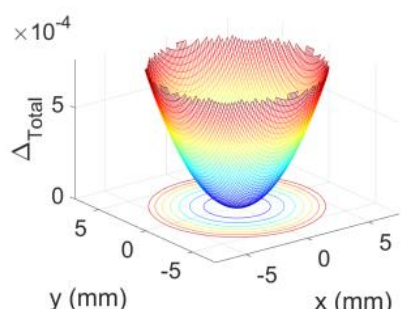

b)

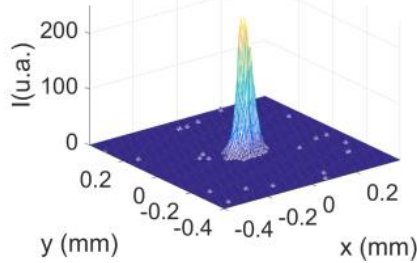

c)

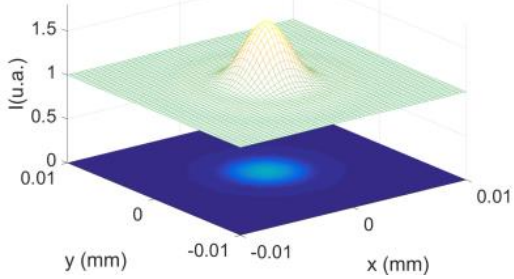

e)

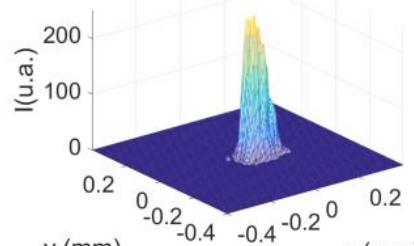

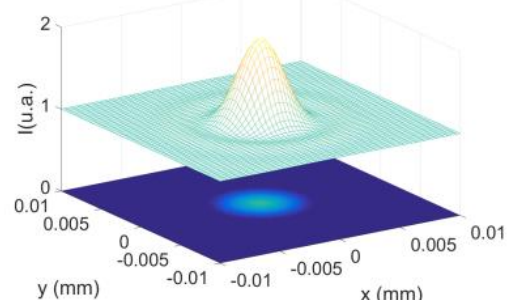

Fig 6. LH's asimétricas negativas reconstruidas con $633 \mathrm{~nm}$ (Fig. 6a, 6b y 6c) y $473 \mathrm{~nm}$ (Fig. 6d, 6e, 6f). Las Fig. 6a y 6d representan la aberración total en el plano de la pupila de salida. Las Fig. 6b y 6e representan los perfiles de intensidad experimentales. Las Fig. 6c y $6 \mathrm{f}$ representan los perfiles de intensidad teóricos normalizados al plano imagen gaussiano.

En el plano de la pupila de salida se observa que en las LH's registradas simétricamente la aberración total se ve afectada principalmente por astigmatismo (Fig. 5a y 5d), mientras que en las LH's con registros asimétricos predomina la aberración esférica (Fig. 6a y 6d). Esto es debido fundamentalmente al tipo de registro (fuera de eje en el primer caso y en eje en el segundo). Al cambiar la $\lambda_{c}$ la $\Delta_{\text {Total }}$ cambia de signo tanto para las LH's simétricas como asimétricas y al reconstruir con $473 \mathrm{~nm}$ la $\Delta_{\text {Total }}$ es un orden de magnitud menor que para $633 \mathrm{~nm}$.

En el plano imagen se puede observar esa tendencia astigmática en el caso de las simétricas de manera experimental (Fig. 5b y 5e) y de manera teórica (Fig. 5c y 5f); y la tendencia esférica en el caso de las asimétricas experimentalmente (Fig. 6b y 6e) y teóricamente (Fig. 6c y 6f). 
a)

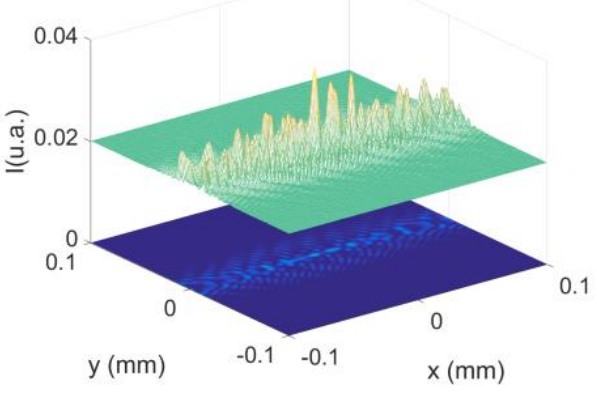

b)

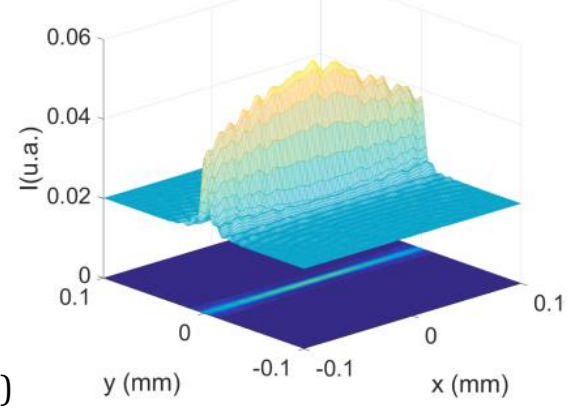

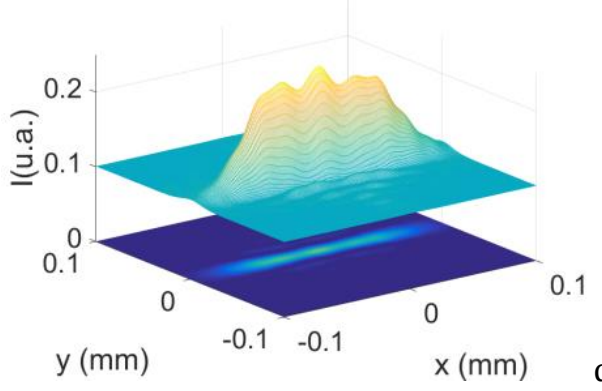

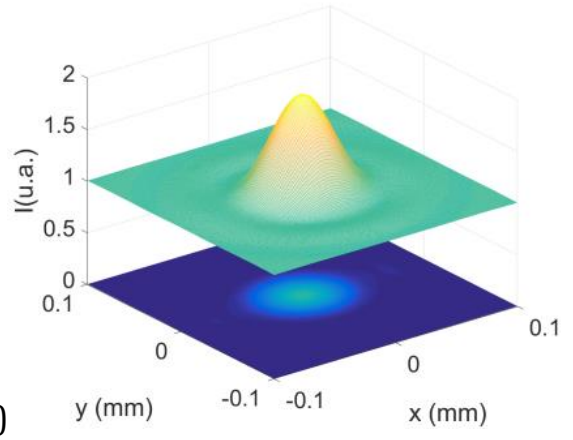

Fig. 7. Compensación de las aberraciones en la LH simétrica positiva $(\lambda=633 \mathrm{~nm})$. a) Radio $=6 \mathrm{~mm}, \mathrm{~b})$ Radio=3mm, c) Radio= $1.5 \mathrm{~mm}$, d) Radio $=0.5 \mathrm{~mm}$.

En resumen, los resultados obtenidos se recogen en la Tabla 2.

TABLA 2. Resultados: Evaluación teórica y experimental de las aberraciones.

\begin{tabular}{|c|c|c|c|c|}
\hline \hline Tipo de LH & $\begin{array}{c}v_{0}(\mathrm{MTF}=0.1) \\
\text { (líneas } / \mathrm{mm})\end{array}$ & $\Delta_{\text {Total }}($ u.a. $)$ & $\begin{array}{c}\text { Tamaño PSF } \\
\left(\mathrm{mm}^{2}\right)\end{array}$ & $I(\%)$ \\
\hline \hline SIM (+) $(633 \mathrm{~nm})$ & 4 & Astigmatismo & $0.9 \times 0.6$ & 0.16 \\
SIM (+) $(473 \mathrm{~nm})$ & 4.9 & Astigmatismo & $0.5 \times 0.6$ & 1.00 \\
SIM (-) $(633 \mathrm{~nm})$ & 5.2 & Astigmatismo & $0.4 \times 0.5$ & 10 \\
SIM (-) $(473 \mathrm{~nm})$ & 10.5 & Astigmatismo & $0.3 \times 0.3$ & 16 \\
\hline ASIM (+) $(633 \mathrm{~nm})$ & 7.4 & Ab. Esférica & $0.2 \times 0.3$ & 70 \\
ASIM (+) $(473 \mathrm{~nm})$ & 8 & Ab. Esférica & $0.2 \times 0.3$ & 100 \\
ASIM (-) $(633 \mathrm{~nm})$ & 17 & Ab. Esférica & $0.2 \times 0.18$ & 70 \\
ASIM (-) $(473 \mathrm{~nm})$ & 12 & Ab. Esférica & $0.2 \times 0.18$ & 100 \\
\hline
\end{tabular}

Para finalizar, se ha propuesto un método para mejorar los perfiles de intensidad de las LH's simétricas positivas. En la Fig. 7 se han representado de manera teórica los distintos perfiles de intensidad de la LH simétrica positiva en función del radio de la pupila de entrada $\left(\phi_{P E} / 2\right)$ para la longitud de onda de $633 \mathrm{~nm}$ manteniendo constante la distancia focal. Se observa claramente que al disminuir el radio a valores más pequeños que el real $(6 \mathrm{~mm})$ las aberraciones van desapareciendo y quedando solamente el perfil de intensidad correspondiente al disco de difracción de Airy, es decir, el perfil imagen gaussiano. En este caso, sólo se producen mejoras que llegan al $71 \%$ de dicha intensidad normalizada. Los datos vienen representados en la Tabla 3. 
TABLA 3. Relación de intensidades normalizadas en función de la apertura numérica del sistema (LH's simétrica positiva).

\begin{tabular}{|c||l|l|l|l|}
\hline Radio (mm) & 6.0 & 3.0 & 1.5 & 0.5 \\
\hline$I(\%)$ & 1.6 & 2.2 & 10 & 71 \\
\hline
\end{tabular}

\section{Conclusiones}

En conclusión, en este trabajo se ha diseñado y optimizado un dispositivo experimental para el almacenamiento de lentes holográficas en un fotopolímero compatible con el medio ambiente, permitiendo registrar LHs positivas y negativas, con registros simétricos y asimétricos. Para evaluar la calidad de imagen de dichas lentes se ha utilizado el cálculo de la MTF de manera teórica y experimental, y se ha realizado una comparación entre el perfil de intensidad en el plano focal imagen experimental (PSF), y el perfil de intensidad teórico en ese mismo plano, es decir, el perfil de intensidad normalizado al plano imagen gaussiano. Además, se ha obtenido de manera cualitativa cómo es la aberración total en el plano de la pupila de salida. Se ha obtenido, por una parte, que tal y como predice la teoría, las aberraciones obtenidas con registros asimétricos, son menores a las obtenidas con registros simétricos, debido fundamentalmente a la gran presencia de astigmatismo, en estos últimos. Por otra parte, se ha estudiado la influencia de la pupila de entrada, y por tanto de la apertura numérica, en la presencia de aberraciones, observando que a menor pupila de entrada (manteniendo constante la distancia focal), las aberraciones disminuyen, por lo que se demuestra que el valor de la apertura numérica es un parámetro fundamental a la hora de fabricar lentes holográficas con mínimas aberraciones.

\section{Agradecimientos}

Este trabajo ha sido subvencionado por el Ministerio de Economía y Competitividad bajo los proyectos FIS2014-56100-C2-1-P, FIS2015-66570-P (MINECO/ FEDER) y FIS2017-82919-R (MINECO/ AEI/ FEDER/ UE), por la Generalitat Valenciana bajo los proyectos PROMETEOII/2015/015, ISIC/2012/013 y y por la Universidad de Alicante bajo la ayuda AII2017-12. 